\title{
Propriedades psicométricas da Frontal Assessment Battery na esclerose múltipla
}

\section{Psychometric properties of the Frontal Assessment Battery in multiple sclerosis}

\author{
Sandra Henriques (1) \\ Helena Espírito-Santo (1,2) \\ Luís Cunha (1) \\ Fernanda Daniel $(1,3)$ \\ Laura Lemos (1)
}

\begin{abstract}
(1) Centro Interdisciplinar de Investigação Psicossocial, Instituto Superior Miguel Torga, Portugal
(2) Centro de Investigação em Neuropsicologia e Intervenção Cognitivo-Comportamental, Faculdade de Psicologia e Ciências da Educação da Universidade de Coimbra, Portugal

(3) Centro de Estudos e Investigação em Saúde da Universidade de Coimbra, Portugal
\end{abstract}

\section{Resumo}

Contexto: A Esclerose Múltipla (EM) é uma doença desmielinizante crónica que pode envolver alterações cognitivas e executivas. As alterações executivas, relacionadas essencialmente com o lobo frontal, podem ser subdiagnosticadas, uma vez que os instrumentos utilizados na EM são extensos e complexos, podendo os seus resultados ser comprometidos pelos níveis de fadiga que poderão daí decorrer. A Bateria de Avaliação Frontal (FAB) é de aplicação rápida e simples e avalia as funções do lobo frontal. Objetivo: Explorar as propriedades psicométricas da FAB numa amostra de doentes com EM. Métodos: No estudo avaliaram-se 68 doentes com EM e 81 indivíduos sem diagnóstico de doença neurológica (amostra de controlo) com a FAB, a Subescala executiva do Montreal Cognitive Assessment/MoCA-E e o Teste de Fluências Verbais Fonéticas/TFVF. Vinte e nove doentes foram reavaliados com a FAB (intervalo 4-8 semanas). Resultados: Na amostra com EM, a consistência interna revelou-se adequada e a estabilidade temporal situou-se entre moderada a alta nas subescalas Semelhanças, FluênciasLexicais, Séries Motoras de Luria e Go-no-Go. A FAB correlacionou-se de forma elevada com o MoCA-E e TFVF, atestando a sua validade convergente, e a sua estabilidade temporal teste-reteste revelou-se adequada. A amostra com EM teve pontuações significativamente inferiores nas subescalas Fluências Lexicais e Séries Motoras de Luria comparativamente com a amostra de controlo $(p<0,05)$. Na EM, a FAB discriminou os níveis de escolaridade $(p<0,001)$ e a subescala Séries Motoras de Luria diferenciou os padrões de EM $(p<0,05)$. Conclusões: A FAB apresenta propriedades psicométricas adequadas para avaliar as funções do lobo frontal em doentes com EM, devendo integrar a sua avaliação neuropsicológica para auxiliar no correto encaminhamento terapêutico. Adicionalmente, a subescala das Fluências Lexicais parece ser importante para avaliar estes doentes, tendo potencial para o nível I do rastreio da disfunção frontal na EM.

Palavras-Chave: Esclerose múltipla; Frontal Assessment Battery; Funcionamento executivo.

$\begin{array}{lll}\text { DI\&D | ISMT } & \text { Publicação em Acesso Aberto } & \text { Helena Espírito Santo } \\ & \text { (c)19.O(s) Autor(es). Esteé um artigo de acesso aberto } & \text { Instituto Superior Miguel Torga } \\ \text { rpics@ismt.pt } & \text { distribuido soba Licença Creative Commons Attribution, que } & \text { Rua Augusta, 46 } \\ \text { https://rpics.ismt.pt } & \text { permite uso, distribuição e reprodução sem restrições em } & \text { 3000-061 Coimbra } \\ & \text { qualquer meio, desde que o trabalho original seja devidamente } & \text { Portugal } \\ \text { helenum@gmail.com }\end{array}$




\begin{abstract}
Background: Multiple Sclerosis (MS) is a chronic demyelinating disease that can involve cognitive and executive changes. Executive changes, mostly related to the frontal lobe, could be underdiagnosed, since the instruments used in MS are extensive and complex, and their results may be compromised by the levels of fatigue that may potentially stem from them. The frontal assessment battery here presented (FAB), can be applied in a fast and easy way, in order to assess frontal lobe functions. Aim: Exploring the psychometric properties of the FAB in a Portuguese sample of MS patients. Method: $A$ sample of $68 \mathrm{MS}$ patients and a control sample $(n=81$ individuals with no diagnosed neurological disease) were assessed with FAB, Montreal Cognitive Assessment Subscale/MoCA-E, and Phonetic Verbal Fluency Test/PVFT. Twenty-nine patients were reassessed with the FAB four-eight weeks after. Results: In the MS sample, internal consistency was adequate, and temporal stability was moderate to high in the Similarities, Lexical Fluency, Luria's Motor Sequences, and GoNo-Go subscales. The FAB results correlated highly with those obtained with both MoCA-E and PVFT, thus assuring adequate convergent validity, and its test-retest temporal stability was adequate. Lexical Fluency and Luria's Motor Sequences subscales scores were significantly lower in MS patients compared with normal controls $(p<.05)$. In MS, FAB discriminated between schooling levels $(p<.001)$, and Luria's Motor Sequences differentiated between MS patterns $(p<$ .05). Conclusions: The FAB presents adequate psychometric properties to assess frontal lobe functions in patients with MS, and it should thus be part of the neuropsychological assessment to help on the correct therapeutic referral. Furthermore, the Lexical Fluency subscale seems important in the assessment of these patients, being potentially a valid measure for MS frontal dysfunction level I screening.
\end{abstract}

Keywords: Executive functioning; Frontal Assessment Battery; Multiple sclerosis.

\title{
Introdução
}

A Esclerose Múltipla (EM) é uma doença crónica, progressiva e desmielinizante do sistema nervoso central que pode conduzir a dificuldades de ordem física, emocional, cognitiva e social (Jougleux-Vie et al., 2014; Keegan \& Noseworthy, 2002). Esta é uma doença autoimune que afeta maioritariamente adultos jovens, apresentando maior incidência no sexo feminino (Keegan \& Noseworthy, 2002; Kingwell et al., 2013; Milo \& Miller, 2014). De acordo com a World Health Organization (2013), estima-se que existam no mundo mais de dois milhões de pessoas com EM. A mesma fonte indica que a Europa regista uma prevalência entre 20 e 60 portadores por cada 100.000 habitantes. Kurtzke (2000) considerou Portugal um país de prevalência alta com aproximadamente $40 / 100.000$ pessoas. Um estudo mais recente (De Sá et al., 2006) encontrou um valor de prevalência aproximado no distrito de Santarém (46,3/100.000 pessoas).

O diagnóstico é, por norma, feito entre os 30 e os 40 anos de idade (Keegan \& Noseworthy, 2002; Kingwell et al., 2013; Milo \& Miller, 2014), baseando-se nas alterações observadas no exame neurológico e comprovadas por estudos laboratoriais, incluindo a análise ao líquido cefalorraquidiano e potenciais evocados ou ressonância magnética (McDonald et al., 2001; Miller, 2001).

A EM é incluída no grupo das doenças degenerativas por envolver a acumulação de défices neurológicos ao longo do tempo (Lezak, Howieson, Bigler, \& Tranel, 2012). O curso clínico da EM pode seguir padrões clínicos muito diversos, mas geralmente caracteriza-se por períodos agudos de agravamento, por deterioração neurológica progressiva ou por uma combinação de ambos (Lublin \& Reingold, 1996; Lublin et al., 2014). Os padrões mais comuns incluem o recidivante-remitente (EM-RR), seguido do secundário progressivo (EM-SP) e do primário progressivo (EM-PP) (Figueiredo, Silva, Cerqueira, Fonseca, \& Pereira, 2015; Jick, Li, Falcone, Vassilev, \& Wallander, 2015; Keegan \& Noseworthy, 2002; Lublin \& Reingold, 
1996; Lublin et al., 2014; Milo \& Miller, 2014). O EM-RR cursa com surtos agudos de alteração neurológica, após o qual o doente recupera totalmente ou fica com sequelas e défices residuais; os períodos entre recaídas caracterizam-se por estabilidade clínica. O padrão EM-SP inicia-se com um curso RR seguido de progressão variável que pode incluir recaídas ocasionais, recuperações menores e períodos de estabilidade. O EM-PP caracteriza-se por um padrão progressivo desde o início com períodos de inatividade ou recuperações variáveis (Lublin \& Reingold, 1996; Lublin et al., 2014).

As principais características clínicas são a destruição da bainha de mielina, a infiltração de células inflamatórias nos espaços perivasculares, o aparecimento de placas e lesões na substância branca e consequente sintomatologia neurológica (Keegan \& Noseworthy, 2002; Milo \& Miller, 2014). Entre os sintomas físicos destacam-se a fraqueza, a rigidez, a descoordenação motora, os défices visuais, as alterações urinárias e intestinais, a disfunção sexual, as alterações sensoriais, a sensibilidade ao calor e a fadiga (Miller, 2001). Outras características comuns da doença são as alterações cognitivas e executivas, podendo ser um dos primeiros indicadores de EM (Amato, Zipoli, \& Portaccio, 2008). O défice de funções cognitivas e executivas está presente em cerca de 45 a 70\% dos doentes (Cotter et al., 2018; DeSousa, Albert, \& Kalman, 2002). O défice cognitivo inclui alterações nos processos atencionais, memória e velocidade de processamento (Amato et al., 2008). Entre as alterações executivas incluem-se os défices no raciocínio abstrato, resolução de problemas, planeamento, monitorização e estimação cognitiva (revisão de Amato et al., 2008). As alterações executivas dizem respeito à interferência com a área préfrontal e suas conexões com outras áreas cerebrais (Alexander \& Stuss, 2000). De facto, um problema comum da EM é a lesão dos lobos frontais (Amato et al., 2008; Roca et al., 2008).

Os défices cognitivo e executivo são mais acentuados nos padrões progressivos da doença onde predomina a componente degenerativa (EM-PP e EM-SP) (Denney, Sworowski, \& Lynch, 2005; Heaton, Nelson, Thompson, Burks, \& Franklin, 1985; Penny, Khaleeli, Cipolotti, Thompson, \& Ron, 2010). No entanto, estes défices são muitas vezes subdiagnosticados devido à falta de instrumentos adequados para avaliar pessoas com EM (Amato et al., 2008; Cotter, et al., 2018). Dois dos instrumentos frequentemente usados para proceder à avaliação neuropsicológica nesta doença são o Questionário de Avaliação Neuropsicológica na EM (MSNQ, Benedict et al., 2004) e a Bateria Repetitiva Breve de Testes Neuropsicológicos (BRBTN; Rao, 1990). Apesar destes instrumentos apresentarem sensibilidade e especificidade adequadas [MSNQ: sensibilidade $83,0 \%$ e especificidade $84,0 \%$; BRBTN: sensibilidade $68 \%$ e especificidade de 85,0\%, (Benedict et al., 2004)], eles são extensos e complexos no que respeita à administração e cotação (Cotter et al., 2018). Os seus resultados podem assim ser comprometidos devido a características clínicas da doença, nomeadamente os níveis de fadiga. Este é um dos sintomas físicos mais incapacitante, com uma prevalência variando entre os 53,0\% e os 83,0\% (Wood et al., 2012).

A Bateria de Avaliação Frontal (FAB) criada por Dubois, Slachevsky, Litvan e Pillon (2000), sendo uma bateria de aplicação rápida e de fácil interpretação, poderá revelar-se útil para colmatar a complexidade e extensão acima mencionada. A FAB é uma bateria de avaliação neuropsicológica que objetiva detetar a disfunção executiva (DE) associada ao lobo frontal através da avaliação de funções como a conceptualização, a flexibilidade mental, a programação motora, a sensibilidade à interferência, o controlo inibitório e a autonomia ambiental (Dubois et al., 2000). A sua administração demora cerca de dez minutos e é constituída por seis subescalas. Desde o estudo original, a FAB apresentou-se como tendo boas propriedades psicométricas (D'Onofrio et al., 2018; Hurtado-Pomares et al., 2018; Kim et al., 
2010; Kugo et al., 2007). Nomeadamente, o estudo de Dubois et al. (2000) indicou um valor de alfa de Cronbach de 0,78, enquanto que o estudo português apresentou um alfa de Cronbach de 0,69 (Lima, Meireles, Fonseca, Castro, \& Garrett, 2008). Ao longo dos anos a FAB tem comprovado as vantagens propostas pelos autores originais ao integrar diversos estudos onde se tem mostrado capaz de discriminar várias patologias neurodegenerativas. Assim, esta bateria permitiu discriminar a doença de Alzheimer (DA) da demência frontotemporal (Slachevsky et al., 2004); a DA da demência vascular (D’Onofrio et al., 2018; Oguro et al., 2006); indivíduos com declínio cognitivo ligeiro (DCL) de indivíduos saudáveis (Chong et al., 2010) e pessoas idosas que sofreram Acidente Vascular Cerebral (AVC) com declínio cognitivo daqueles sem declínio (Espirito-Santo, Garcia, Monteiro, Carolino, \& Daniel, 2016). Vários trabalhos validaram a FAB na doença de Parkinson (DPa) (Bezdicek et al., 2017; Bugalho \& Vale 2011; Hurtado-Pomares et al., 2018; Matsui et al., 2006). Adicionalmente, a FAB tem sido alvo de validação e adaptação em vários países com várias populações (Appollonio et al., 2005; Asaadi et al., 2016; Benke, Karner, \& Delazer, 2013; Kim et al., 2010; Kugo et al., 2007; Wang, Hung, \& Yang, 2015). No que respeita ao uso desta bateria na EM, vários estudos utilizaram-na como parte dos seus instrumentos de avaliação; no entanto, nenhum desses estudos constituiu o exame das propriedades psicométricas da FAB para esta população (Chan et al., 2017; Felippe, Salgado, Silvestre, Santos, \& Christofoletti, 2018; Jougleux-Vie et al., 2014; Montel \& Burgener, 2007; Raimo et al., 2014; Roca et al., 2008, 2014).

Como os doentes com EM são suscetíveis ao défice das FE e cognitivas e esse défice pode, por vezes, ser subdiagnosticado devido à falta de instrumentos adequados e validados, importa rastrear a DE nesta população, tanto para auxiliar num diagnóstico correto que permita um encaminhamento terapêutico adequado, como para servir de base a novas terapias de reabilitação neurocognitiva. Uma vez que a FAB se tem revelado adequada e sensível para usar em diversas patologias neurodegenerativas, o presente trabalho objetiva analisar as propriedades psicométricas (consistência interna, estabilidade temporal e validade convergente) desta bateria para uso na população portuguesa de doentes com EM. Pretende-se também analisar a capacidade da FAB para discriminar uma subamostra de doentes com EM de uma subamostra de pessoas da comunidade sem doenças neurológicas diagnosticadas, bem com analisar as diferenças individuais na FAB.

\section{Métodos}

\section{Participantes}

A Tabela 1 apresenta as características sociodemográficas e clínicas da amostra. Para um melhor tratamento dos dados sociodemográficos e clínicos, algumas variáveis foram agrupadas e recodificadas. Assim, a idade foi dividida em quatro subgrupos (30-40, 41-50, 51-60 e > 61 anos), seguindo a divisão feita em alguns dos estudos de validação analisados (Appollonio et al., 2005; Espirito-Santo et al., 2016; Lima et al., 2008). As profissões foram recodificadas em manuais e intelectuais, de acordo com a classificação de Sohn-Rethel e Sohn-Rethel (1978). A variável surtos de EM foi dividida em seis subgrupos (Nenhum, 1-5, 6-10, 11-15, 16-20, 21-25 surtos), tendo em conta um estudo relativo à prevalência de surtos (Negreiros, Sousa-Munõz, Oliveira, Nóbrega, \& Monteiro, 2015). 
Assim, observa-se que a amostra global ficou constituída por 149 participantes sendo 46 do sexo masculino e 103 do sexo feminino. A idade média situou-se nos 47,72 ( $D P=11,42$ ), a escolaridade média nos $12,41(D P=4,71)$ anos e $81,2 \%$ desempenhavam profissões intelectuais. A subamostra clínica (SAC) foi constituída por 68 participantes ( 21 do sexo masculino e 47 do sexo feminino), com uma idade média de 48,03 ( $D P=10,51)$ e uma escolaridade de $12,29(D P=4,80)$ anos. A subamostra não-clínica (SANC) contou com 81 participantes ( 25 do sexo masculino e 56 do sexo feminino), com uma idade média de $47,46(D P=12,20)$ anos e uma escolaridade de $12,51(D P=4,66)$ anos. Não se verificaram diferenças estatisticamente significativas entre as subamostras em nenhuma das variáveis mencionadas. A subamostra clínica (SAC), foi maioritariamente composta pelo padrão EM-RR (54,4\%), seguido do EM-SP $(22,1 \%)$ e do EM-PP (10,3\%), 41,2\% dos participantes diz ter vivenciado entre 1 a 5 surtos. A idade média de diagnóstico situou-se nos 33 anos ( $D P=11,10)$, sendo que a duração da doença apresentou uma média de 176,10 meses ( $D P=116,20$; amplitude: 470; 7 - 477 meses). Os participantes apresentavam uma incapacidade média atribuída (de acordo com atestado médico) de 70,80 ( $D P=16,20)$ e tomavam em média 4,08 fármacos ( $D P=2,90 ; M o=1$; amplitude: 14; 0 - 14 fármacos).

\section{Tabela 1}

Caracterização Sociodemográfica da Amostra ( $\mathrm{N}=149)$

\begin{tabular}{|c|c|c|c|c|c|c|c|c|}
\hline & & \multicolumn{2}{|c|}{ AG } & \multicolumn{2}{|c|}{$\operatorname{SAC}(n=68)$} & \multicolumn{2}{|c|}{ SANC $(n=81)$} & \multirow{2}{*}{$\chi^{2}$} \\
\hline & & $n$ & $\%$ & $n$ & $\%$ & $n$ & $\%$ & \\
\hline \multirow{2}{*}{ Sexo } & Masculino & 46 & 30,9 & 21 & 45,7 & 25 & 54,3 & \multirow{2}{*}{$\approx 0,00^{\text {ns }}$} \\
\hline & Feminino & 103 & 69,1 & 47 & 45,6 & 56 & 54,4 & \\
\hline \multirow{4}{*}{$\begin{array}{l}\text { Idade } \\
\text { AG: } M=47,72 \pm 11,42 \\
\text { SAC: } M=48,03 \pm 10,51 \\
\text { SANC: } M=47,46 \pm 12,20 \\
t=0,30 ; p=0,762\end{array}$} & $30-40$ & 43 & 28,9 & 15 & 34,9 & 28 & 65,1 & \multirow{4}{*}{$5,86^{\text {ns }}$} \\
\hline & $41-50$ & 48 & 32,2 & 28 & 58,3 & 20 & 41,7 & \\
\hline & $51-60$ & 41 & 27,5 & 19 & 46,3 & 22 & 53,7 & \\
\hline & $>61$ & 17 & 11,4 & 6 & 35,3 & 11 & 64,7 & \\
\hline \multirow{7}{*}{$\begin{array}{l}\text { Habilitações Literárias } \\
\text { AG: } M=12,41 \pm 4,71 \\
\text { SAC: } M=12,29 ; \pm 4,80 \\
\text { SANC: } M=12,51 ; \pm 4,66 \\
t=0,32 ; p=0,747\end{array}$} & Sabe ler e escrever ${ }^{1}$ & 2 & 1,3 & 0 & 0,0 & 2 & 100,0 & \multirow{7}{*}{$0,11^{\text {nsa }}$} \\
\hline & $1^{\circ}$ ciclo & 11 & 7,4 & 6 & 54,5 & 5 & 45,5 & \\
\hline & $2^{\circ}$ ciclo & 7 & 4,7 & 4 & 57,1 & 3 & 42,9 & \\
\hline & $3^{\circ}$ ciclo & 27 & 18,1 & 13 & 48,1 & 14 & 51,9 & \\
\hline & Ensino Secundário & 49 & 32,9 & 21 & 42,9 & 28 & 57,1 & \\
\hline & Licenciatura & 40 & 26,8 & 19 & 47,5 & 21 & 52,5 & \\
\hline & Mestrado & 13 & 8,7 & 5 & 38,5 & 8 & 61,5 & \\
\hline \multirow{2}{*}{ Categoria Profissional } & Manual & 28 & 18,8 & 12 & 42,9 & 16 & 57,1 & \multirow{2}{*}{$0,11^{\mathrm{ns}}$} \\
\hline & Intelectual & 121 & 81,2 & 56 & 46,3 & 65 & 53,7 & \\
\hline
\end{tabular}

Nota AG = Amostra Global; $S A C=$ Subamostra Clínica; SANC = Subamostra não-clínica; $\chi^{2}=$ Qui-quadrado da independência; $M=$ Média; $N=$ Número de participantes; ${ }^{1}$ Sem escolaridade.

${ }^{a}$ Associação linear-por-linear.

${ }^{n}$ Não significativo. 
Para critérios de inclusão estabeleceu-se ser falante de língua Portuguesa e residir em Portugal. Para a SAC foi ainda critério essencial de inclusão, a existência de um diagnóstico de EM devidamente comprovado, independentemente da tipologia da doença. Excluíram-se utentes que possuíssem alguma incapacidade física visível (problemas de visão, audição, limitações motoras ou afasias), que impedisse a participação nas provas exigidas, ou doença mental grave ou qualquer outra doença neurológica, tal como constava nos registos clínicos existentes nas delegações da SPEM. Ao contrário do que se verificou com Bezdicek et al. (2017), não foram excluídos participantes com défice cognitivo e/ou sintomas depressivos por se considerar, de acordo com outros trabalhos (Amato et al., 2008; Siegert \& Abernethy, 2005), que estas características são representativas da população em estudo.

\section{Instrumentos}

Foram utilizados três testes para medir as principais funções executivas. O Teste de Fluência Verbal Fonética e a subescala executiva do MoCA foram usados para analisar a validade convergente da FAB.

A Bateria de Avaliação Frontal, objeto de estudo da presente investigação, é um instrumento de rastreio que avalia as funções associadas ao lobo frontal. A prova inicia-se pela avaliação do pensamento abstrato através da subescala das Semelhanças. A avaliação da flexibilidade mental é feita através da Fluência Lexical. Na terceira subescala avalia-se a programação motora pelas Séries Motoras de Luria. A subescala das Instruções Antagónicas visa avaliar a sensibilidade à interferência. A quinta subescala denominada Gono-Go objetiva avaliar o controlo inibitório e termina-se com a subescala do Comportamento de Preensão para avaliar a autonomia ambiental. Quanto à pontuação, cada subescala é cotada numa escala de 0 a $3 \mathrm{e}$ a pontuação total varia entre 0 e 18 pontos (Dubois et al., 2000).

O Teste de Fluência Verbal Fonética (TFVF, versão original de Benton \& Hamsher, 1978; tradução e validação portuguesa de Sarmento, 2018) foi desenvolvido por Thurstone em 1938. Este é um teste neuropsicológico que objetiva avaliar a linguagem, a memória e as FE. Existem várias versões no que toca às letras utilizadas. Em Portugal um estudo mostrou que as letras que melhor discriminam a existência de défice cognitivo são o $P$, o U e o V (Sarmento, 2018) e esta foi a versão utilizada neste estudo. Relativamente ao valor de confiabilidade, os valores de alfa de Cronbach das versões portuguesas foram de 0,89 (Braz, 2017) e de 0,80 (Sarmento, 2018). No presente estudo o alfa de Cronbach encontrado foi de 0,75 .

O Montreal Cognitive Assessment (MoCA; versão original de Nasreddine et al., 2005; tradução e validação portuguesa de Simões et al., 2008) é um instrumento que avalia o défice cognitivo através das aptidões visuoespaciais, $\mathrm{FE}$, memória, atenção, concentração, memória de trabalho, linguagem e orientação. $A$ pontuação máxima deste instrumento é de 30 pontos (Nasreddine et al., 2005; Simões et al., 2008). No que diz respeito à consistência interna, a versão portuguesa apresentou um valor de alfa de Cronbach de 0,71 (Freitas, Simões, Martins, Vilar, \& Santana, 2010). O presente estudo indicou um valor de 0,83.

\section{Procedimentos}

O presente estudo, de caráter preliminar, integrou o projeto "Trajetórias de Saúde mental em doentes com Esclerose Múltipla", que tem como objetivo proceder à caracterização da população portuguesa de 
doentes com EM relativamente a variáveis de natureza psicológica, neuropsicológica e física. É igualmente propósito deste projeto criar e validar um conjunto de instrumentos neuropsicológicos para avaliar a população portuguesa portadora de EM. Para a presente investigação, contámos com as delegações da Sociedade Portuguesa de EM (SPEM) de quatro cidades portuguesas do centro e litoral de Portugal continental.

Todos os participantes que aceitaram participar no estudo foram informados acerca dos objetivos da investigação e o consentimento informado foi assinado após a natureza voluntária da cooperação ter sido enfatizada. Cinco participantes recusaram participar no estudo $(3,2 \%)$ e dois $(1,3 \%)$ foram excluídos devido à existência de limitações motoras e afasias. Por forma a cumprir com os pressupostos éticos e deontológicos inerentes à investigação com seres humanos, a presente investigação foi analisada e aprovada pela Comissão de Ética do ISMT.

A recolha de dados decorreu entre dezembro de 2018 e maio de 2019 nas instalações da SPEM ou na residência dos participantes (membros associados da SPEM). A maioria dos participantes da SAC foi contactada pelos responsáveis das delegações da SPEM. A SANC foi recrutada na esfera social das investigadoras (familiares, amigos e colegas), através de amostragem por conveniência e foi sujeita aos mesmos instrumentos de avaliação. Alguns participantes da SAC $(n=29)$ foram reavaliados com um intervalo de tempo de quatro a oito semanas, tendo em conta a disponibilidade dos participantes e a sua localização geográfica, de modo a analisar a estabilidade temporal dos instrumentos.

\section{Análise Estatística}

A análise e o tratamento dos dados foram feitos com recurso ao Programa Estatístico Statistical Package for the Social Sciences (IBM SPSS Statistics, versão 25.0 para Macintosh, 2012).

Para analisar as características sociodemográficas e clínicas das subamostras, usou-se o teste de Qui Quadrado da independência e o Qui Quadrado da aderência com Análise de Monte Carlo. A simulação de Monte Carlo tem por base a geração aleatória de amostras e usa-se quando existem classes com um número reduzido de elementos (Marôco, 2014).

Foi verificada a normalidade da distribuição das pontuações de todos os instrumentos, através do teste de Shapiro-Wilk e sempre que houvesse indicação de não-normalidade $(p<0,05)$, calculou-se a simetria e a curtose seguindo as diretrizes de Kim (2013) (valor $z$ absoluto abaixo de 3,29 da curtose e da assimetria são indicadores de normalidade para amostras superiores a 50 e inferiores a 300 participantes).

Foram realizadas as análises descritivas (médias, desvio-padrão, frequências, mínimo e máximo) para a FAB e respetivas subescalas. Quanto às propriedades psicométricas, recorreu-se à análise do alfa de Cronbach, correlações inter-item e correlações item total para analisar a confiabilidade (Daniel, Gomes, \& Ferreira, 2015). As correlações de Spearman para a pontuação total e as correlações bisserial para as subescalas foram usadas para a análise da estabilidade temporal (Daniel et al., 2015). A validade convergente foi analisada recorrendo ao cálculo das correlações de Spearman.

Para a determinação da capacidade discriminativa, efetuou-se a análise das diferenças individuais na FAB com recurso ao teste $t$ de Student para amostras independentes, $U$ de Mann-Whitney ou ANOVA 
(Marôco, 2014), conforme o pressuposto da normalidade, requerido pelos testes paramétricos, fosse verificado ou não.

A determinação dos correlatos da FAB foi feita através da análise correlacional de Pearson ou de Spearman observando o mesmo critério e de acordo com as indicações de Marôco (2014).

Para cada teste estatístico, o nível de significância foi estabelecido num valor mínimo de alfa de 0,05 e calculado o respetivo tamanho do efeito [ $d$ de Cohen ou g de Hedges, fórmula do tamanho de efeito para o teste U de Mann-Whitney, Eta Quadrado para ANOVA e coeficiente de determinação $\left(r^{2} \times 100\right)$ ] seguindo as indicações de Espirito-Santo e Daniel (2015, 2017, 2018).

Considerando o tamanho da amostra, determinámos o poder estatístico para cada teste. Assim, para o Qui Quadrado da Aderência o poder foi de 89,40\% para um tamanho do efeito de 0,43 e alfa de 0,05 (cálculo com o G*Power sg. Faul, Erdfelder, Lang, \& Buchner, 2007). Para o teste t de Student ou U de Mann-Whitney obteve-se um poder superior a $95 \%$, tendo em conta um alfa a 5,0\%, as médias e desvios-padrão da $\mathrm{FAB}$ na SAC, na SANC e a taxa de amostragem $\left(\kappa=n_{\mathrm{sac}} / n_{\mathrm{sanc}}=0,63\right)$ (cálculos através de calculadora de HyLown Consulting, 2013-2019). Para a ANOVA, considerando o tamanho da amostra global (AG; $N=$ 149), um tamanho de efeito médio ( $f=0,25 ; r=0,5$ ) e um alfa de 0,05 , o poder variou entre 58,0 e $78,0 \%$. Quanto à correlação, considerando um tamanho de efeito médio $(r=0,3)$, o poder estatístico foi superior a 95,0\%. No que concerne à estabilidade temporal, considerando o valor das correlações encontrados na revisão da literatura (Appollonio et al., 2005; Hurtado-Pomares et al., 2018) e o número de participantes que foram reavaliados $(n=29)$, o poder estatístico para a analise correlacional variou entre $87,4 \%$ a $100 \%$ (cálculos com o G*Power; Faul et al., 2007).

\section{Resultados}

\section{Análises preliminares}

Análise de normalidade. Para a decisão estatística procedeu-se à análise de normalidade de distribuição das pontuações da FAB e dos restantes testes neuropsicológicos. Assim, os valores $z$ da simetria e curtose, de acordo com Kim (2013), indicam uma distribuição não normal das pontuações totais do FAB em ambas as subamostras (SAC, $s i=-3,89 ; c u=1,34$. SANC, $s i=4,34 ; c u=0,87$ ) e das suas subescalas (SAC, si entre -11,36 e -2,30; cu entre 19,74 e 0,96. SANC, si entre -12,51 e -4,10; cu entre 23,19 e 0,87). Quanto aos restantes dois testes, as distribuições foram diversas em termos da normalidade [MoCA-E (SAC, si =-2,99; $c u=0,42$; SANC, $s i=-3,93 ; c u=2,09$ ) e TFVF (SAC, $s i=0,89 ; c u=0,67$; SANC, si = 0,47; $c u=1,27)]$.

Descritivas. Na Tabela 2 pode verificar-se que a média total da FAB para a SAC foi $16,00(D P=2,16)$ e para a SANC foi de $16,63(D P=1,62)$. 
Tabela 2

Análises Descritivas e das Diferenças da Bateria de Avaliação Frontal e Respetivas Subescalas, do Montreal Cognitive Assessement-Subescala Executiva e do Teste de Fluências Verbais Fonéticas ( $N=149)$

\begin{tabular}{|c|c|c|c|c|c|c|c|c|c|}
\hline \multirow{2}{*}{\multicolumn{2}{|c|}{ Subescalas }} & \multicolumn{3}{|c|}{$\operatorname{SAC}(n=68)$} & \multicolumn{3}{|c|}{ SANC $(n=81)$} & \multirow{2}{*}{$U / t$} & \multirow{2}{*}{$\begin{array}{c}\text { gde } \\
\text { Hedges }\end{array}$} \\
\hline & & $M \pm D P$ & Mín & Máx & $M \pm D P$ & Mín & Máx & & \\
\hline \multicolumn{2}{|c|}{ FAB Total } & $16,00 \pm 2,16$ & 9,00 & 18,00 & $16,63 \pm 1,62$ & 12,00 & 18,00 & $1,98^{*}$ & $0,33^{\dagger}$ \\
\hline \multicolumn{2}{|c|}{ Semelhanças } & $2,53 \pm 0,80$ & 0,00 & 3,00 & $2,68 \pm 0,50$ & 1,00 & 3,00 & $2650,50^{\text {ns }}$ & $0,23^{\dagger}$ \\
\hline \multicolumn{2}{|c|}{ Fluência Lexical } & $2,37 \pm 0,71$ & 1,00 & 3,00 & $2,70 \pm 0,68$ & 0,00 & 3,00 & $1960,00^{* * *}$ & $0,48^{\dagger}$ \\
\hline \multicolumn{2}{|c|}{ Séries Motoras de Luria } & $2,59 \pm 0,71$ & 1,00 & 3,00 & $2,81 \pm 0,52$ & 0,00 & 3,00 & $2342,00^{*}$ & $0,36^{\dagger}$ \\
\hline \multicolumn{2}{|c|}{ Instruções Antagónicas } & $2,88 \pm 0,37$ & 1,00 & 3,00 & $2,83 \pm 0,38$ & 2,00 & 3,00 & $2568,50^{\text {ns }}$ & $0,13^{\dagger}$ \\
\hline \multicolumn{2}{|c|}{ Go-no-Go } & $2,63 \pm 0,69$ & 0,00 & 3,00 & $2,60 \pm 0,70$ & 0,00 & 3,00 & $2699,00^{\text {ns }}$ & $0,04^{\text {ns }}$ \\
\hline \multicolumn{2}{|c|}{ Comportamento de Preensão } & $3,00 \pm 0,00$ & 3,00 & 3,00 & $3,00 \pm 0,00$ & 3,00 & 3,00 & $2754,00^{\text {ns }}$ & 0,00 \\
\hline \multicolumn{2}{|c|}{ MoCA-E } & $2,93 \pm 1,06$ & 0,00 & 4,00 & $3,19 \pm 0,88$ & 0,00 & 4,00 & $2402,50^{\text {ns }}$ & 0,00 \\
\hline \multirow{3}{*}{ TFVF } & $P$ & $10,34 \pm 4,20$ & 3,00 & 2,00 & $12,12 \pm 4,60$ & 1,00 & 29,00 & $2,46^{\mathrm{ns}}$ & $0,40^{\dagger}$ \\
\hline & $\mathbf{U}$ & $4,53 \pm 2,46$ & 0,00 & 1,00 & $5,17 \pm 2,93$ & 0,00 & 15,00 & $1,43^{\text {ns }}$ & $0,23^{\dagger}$ \\
\hline & v & $7,75 \pm 3,15$ & 2,00 & 16,00 & $8,68 \pm 3,15$ & 1,00 & 16,00 & $1,79^{\text {ns }}$ & $0,30^{\dagger}$ \\
\hline
\end{tabular}

Nota. $M$ = Média; $D P$ = Desvio-padrão; Máx = Máximo; Mín = Mínimo; U = Teste U de Mann-Whitney; $t$ = Teste $t$ de Student; $S A C=S u b a m o s t r a$ Clínica; SANC = Subamostra não-clínica; FAB = Bateria de Avaliação Frontal; MoCA-E = Montreal Cognitive Assessement- subescala executiva; TFVF = Teste de Fluências Verbais Fonéticas.

a Tempo de nomeação.

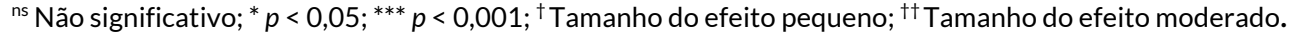

Na Tabela 3 encontram-se as frequências das pontuações em cada subescala da FAB. É possível observar que a SAC teve, de forma estatisticamente significativa, mais pontuações inferiores (inferior a 3 pontos), nas subescalas da Fluência lexical e das Séries Motoras de Luria. A subescala Comportamento de Preensão foi pontuada no máximo por todos os participantes de ambas as subamostras.

\section{Tabela 3}

Distribuição de Frequências das Pontuações nas Subescalas da Bateria de Avaliação Frontal por Subamostra Clínica (SAC: $\mathrm{n}=68$ ) e Subamostra Não-clínica (SANC: $\mathrm{n}=81$ )

\begin{tabular}{|c|c|c|c|c|c|c|c|c|c|c|c|c|c|}
\hline & & \multicolumn{2}{|c|}{ SM \% } & \multicolumn{2}{|c|}{ FL \% } & \multicolumn{2}{|c|}{ SML \% } & \multicolumn{2}{|c|}{ IA \% } & \multicolumn{2}{|c|}{ GNG \% } & \multicolumn{2}{|c|}{ CP \% } \\
\hline & & SAC & SANC & SAC & SANC & SAC & SANC & SAC & SANC & SAC & SANC & SAC & SANC \\
\hline Mediana & & 3,00 & 3,00 & 2,50 & 3,00 & 3,00 & 3,00 & 3,00 & 3,00 & 3,00 & 3,00 & 3,00 & 3,00 \\
\hline \multirow{4}{*}{ Pontuações } & 0 & 2,9 & 0,0 & 0,0 & 2.5 & 0,0 & 1.2 & 0,0 & 0,0 & 1,5 & 1,2 & 0,0 & 0,0 \\
\hline & 1 & 10,3 & 1,2 & 13.2 & 4.9 & 13.2 & 2.5 & 1.5 & 0,0 & 7,4 & 8,6 & 0,0 & 0,0 \\
\hline & 2 & 17,6 & 29,6 & 36.8 & 12.3 & 14.7 & 9.9 & 8.8 & 17.3 & 17,6 & 18,5 & 0,0 & 0,0 \\
\hline & 3 & 69,1 & 69,1 & 50.0 & 80.2 & 72.1 & 86.4 & 89.7 & 82.7 & 73,5 & 71,6 & 100,0 & 100,0 \\
\hline$\chi^{2}$ & & \multicolumn{2}{|c|}{$1,93^{\text {ns }}$} & \multicolumn{2}{|c|}{$8,25^{* *}$} & \multicolumn{2}{|c|}{$4,79^{*}$} & \multicolumn{2}{|c|}{$0,80^{\text {ns }}$} & \multicolumn{2}{|c|}{$0,06^{\mathrm{ns}}$} & \\
\hline
\end{tabular}

Nota. $\chi^{2}=$ Associação linear-por-linear. SM = Semelhanças; FL = Fluência lexical; SML = Séries Motoras de Luria; IA = Instruções Antagónicas; GNG = Go-No-Go; CP = Comportamento de Preensão.

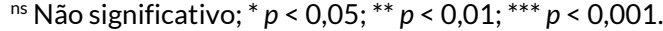




\section{Propriedades psicométricas}

Dado o objetivo principal do presente estudo, as propriedades psicométricas foram analisadas somente para a SAC.

Consistência interna. O alfa de Cronbach revelou um valor de 0,64. Este valor indica uma consistência interna baixa (Daniel et al., 2015; Peterson, 1994), ainda que a confiabilidade se tenha mostrado moderada em quatro subescalas, com correlações item-total superiores a 0,30 (Tabela 4). O valor de alfa foi calculado com cinco itens, ou seja, sem a subescala Comportamento de Preensão que, por possuir variância zero, foi automaticamente eliminada. Relativamente às Instruções Antagónicas, embora este item não atingisse uma correlação item-total superior a 0,30 (Marôco, 2014), foi mantido nas análises, pois ao eliminá-lo a consistência interna da FAB mantinha-se igual.

\section{Tabela 4}

Análise de Confiabilidade das Subescalas da Bateria de Avaliação Frontal ( $\mathrm{N}=68)$

\begin{tabular}{lcc}
\hline Subescalas & $r$ item-total corrigida & Alfa se item excluído \\
\hline Semelhanças & 0,35 & 0,61 \\
Fluência Lexical & 0,49 & 0,54 \\
Séries Motoras de Luria & 0,43 & 0,57 \\
Instruções Antagónicas & 0,26 & 0,64 \\
Go-no-Go & 0,46 & 0,55 \\
\hline
\end{tabular}

Nota. $r=$ Correlação

Estabilidade temporal. $\mathrm{A}$ análise teste-reteste para a $\mathrm{FAB}$-total revelou um valor moderado $\left(r=0,36 ; r^{2}=\right.$ $12,96 \%)$ com tendência à significância estatística $(p=0,058)$ (Espirito-Santo \& Daniel, 2017). No entanto, as correlações foram altas nas subescalas das Semelhanças $(r=0,56 ; p<0,01)$ e da Fluência Lexical $(r=$ 0,50; $p<0,01)$ e moderada nas Séries Motoras de Luria ( $r=0,44 ; p<0,05)$ (Espirito-Santo \& Daniel, 2017). As restantes subescalas apresentaram correlações moderadas e baixas (Espirito-Santo \& Daniel, 2017), não significativas (Go-no-Go: $r=0,31 ; p>0,05$; Instruções Antagónicas: $r=0,10 ; p>0,05$ ) e a análise correlacional não foi calculada para o Comportamento de Preensão devido à variância zero.

Validade convergente. Como se pode observar na Tabela 5, a FAB-Total apresentou correlações elevadas (Espirito-Santo e Daniel, 2017) com o MoCA-E e o TFVF. No que respeita às subescalas da FAB, registaram-se: correlações elevadas entre a Fluência Lexical e o MoCA-E e a Fluência Lexical e o TFVF. 


\section{Tabela 5}

Correlações de Spearman entre a Bateria de Avaliação Frontal, o Montreal Cognitive Assessement - subescala executiva (MoCA-E) e o Teste de Fluências Verbais Fonéticas (TFVF) $(n=68)$

\begin{tabular}{lcc}
\hline & MoCA-E & TFVF \\
\hline FAB Total & $0,58^{* * *}$ & $0,63^{* * *}$ \\
Semelhanças & $0,40^{* * *}$ & $0,31^{* * *}$ \\
Fluência Lexical & $0,58^{* * *}$ & $0,65^{* * *}$ \\
Séries Motoras de Luria & $0,32^{* * *}$ & $0,35^{* * *}$ \\
Instruções Antagónicas & $0,19^{*}$ & $0,13^{\text {ns }}$ \\
Go-No-Go & $0,25^{* *}$ & $0,36^{* * *}$ \\
Comportamento de Preensão & - & - \\
\hline
\end{tabular}

Nota. A negrito as correlações elevadas.

${ }^{*} p<0,05 ;{ }^{* *} p<0,01 ;{ }^{* * *} p<0,001 ;{ }^{\text {ns }}$ Não significativo.

\section{Capacidade discriminativa}

$\mathrm{Na}$ Tabela 2 verifica-se que as pontuações da FAB discriminaram de forma moderada e estatisticamente significativa a SAC da SANC $\left(t=1,98 ; p<0,05 ; g=0,33^{\dagger}\right)$. Quanto às subescalas, somente a Fluência Lexical $\left(U=1960,00 ; p<0,001 ; g=0,48^{\dagger}\right)$ e as Séries Motoras de Luria $\left(U=2342,00 ; p<0,05 ; g=0,36^{\dagger}\right)$ discriminaram as duas amostras.

\section{Diferenças individuais na SAC}

Variáveis sociodemográficas. $O$ desempenho na $\mathrm{FAB}$ variou somente entre os níveis de escolaridade $(F=$ $5,46 ; p<0,001)$, com as pontuações mais baixas no primeiro ciclo $(M=13,50 \pm 2,17$. Os resultados não variaram em função do sexo, idade e categoria profissional.

Variáveis clínicas. No que concerne às tipologias de EM, a ANOVA mostrou que houve diferenças estatisticamente significativas apenas na subescala Séries Motoras de Luria $(F=4,95 ; p<0,05)$. As comparações post-hoc de Games-Howell mostraram que as pontuações nas Séries Motoras de Luria foram significativamente inferiores $(p<0,05)$ no $\operatorname{EM-SP}(M=2,13 \pm 0,92)$ e no $\operatorname{EM}-\operatorname{RR}(M=2,68 \pm 0,63)$ quando comparadas com o EM-PP $(M=3,00 \pm 0,00)$. Ao considerar-se a correção de Bonferroni para o valor do $p(0,05$ / 3 comparações $=0,0167$ ), as diferenças mantiveram-se estatisticamente significativas. Os resultados da FAB não se correlacionaram com o número total de surtos, com o número total de fármacos, com a idade em que foi feito o diagnóstico, nem com a percentagem de incapacidade atribuída. A única correlação com o número de meses desde o diagnóstico ocorreu com as Séries Motoras de Luria $(r=-0,36 ; p<0,05)$. 


\section{Discussão}

A presente investigação teve como objetivo estudar o comportamento da FAB numa amostra de doentes com EM.

A média encontrada na amostra clínica aproxima-se da encontrada por Chan et al. (2017) com doentes com EM ( $M=16,30 \pm 2,04)$ e é superior aos resultados de um estudo com pacientes com demência inicial (e.g., Chong al., 2010; $M=9,74 \pm 3,50$ ). No entanto, para além de as patologias não serem as mesmas, as características sociodemográficas podem também explicar as pontuações mais altas na presente amostra, pois esta é mais jovem e mais escolarizada. Outra explicação é o facto da amostra em estudo incluir maioritariamente doentes com EM-RR que constitui o padrão mais prevalente (Figueiredo et al., 2015; Jick et al., 2015; Keegan \& Noseworthy, 2002; Milo \& Miller, 2014), mas também o que apresenta menos défices executivo e cognitivo (Denney et al., 2005; Heaton et al., 1985; Smestad, Sandvik, Landrø, \& Celius, 2010).

Os doentes com EM tiveram de forma estatisticamente significativa mais pontuações inferiores nas subescalas das Fluência lexical - resultado que é similar aos das investigações de Appollonio et al. (2005), Hurtado-Pomares et al. (2018), Lima et al. (2008) - e nas Séries Motoras de Luria, tal como em Asaadi et al. (2016). Voltaremos a estes pontos mais em baixo.

A subescala Comportamento de Preensão foi pontuada no máximo por todos os participantes, tal como aconteceu em Appollonio et al. (2005) e Lima et al. (2008). Alguns autores sugerem que esta subescala deveria ser substituída por outro teste executivo breve uma vez que, por representar um reflexo primitivo, só apresentará pontuações baixas em pacientes com afeção frontal grave (Lagarde et al., 2013; Mok et al., 2004).

A consistência interna é relativamente baixa, mas tendo em consideração as correlações item-total entre moderadas a elevadas em quatro subescalas, a FAB tende para uma consistência interna adequada. Valores de alfa de Cronbach similares foram encontrados por Bezdicek et al. (2017, doentes DPa: $\alpha$ de Cronbach $=0,60$ ) e Benke et al. (2013, idosos cognitivamente saudáveis: $\alpha$ de Cronbach $=0,46)$. Uma consistência interna baixa pode indiciar que as subescalas medem funções executivas de forma independente (Benke et al., 2013) e, provavelmente, a FAB, mais do que fornecer informação sobre o estado global das funções frontais, poderá assim sugerir a possível localização de lesões frontais.

A estabilidade temporal mostra-se razoável com correlações entre moderadas e elevadas nas subescalas, mas ao contrário do que se verificou em estudos de validação prévios, onde a estabilidade temporal foi elevada (Appollonio et al., 2005; Asaadi et al., 2016; Hurtado-Pomares et al., 2018; Kim et al., 2010; Kugo et al., 2007; Wang et al., 2015). No entanto, nenhum desses estudos se referiu a sujeitos com EM, sendo então necessário ter em conta que esta doença pode acompanhar-se de flutuações no funcionamento cognitivo e executivo em períodos curtos de tempo. Acresce que a presença de dor e de fadiga, tipicamente presentes na EM, podem condicionar o funcionamento geral do sujeito (Kratz et al., 2017; Powell, Liossi, Schlatz, \& Moss-Morris, 2017). Por fim, de acordo com Rabbitt (1997), medidas do funcionamento executivo podem ser limitadas pela sua própria confiabilidade, uma vez que apenas tarefas novas podem detetar a disfunção executiva. Portanto, ao realizar-se a reavaliação a tarefa deixa de ser nova. 
A FAB correlaciona-se de forma elevada com o MoCA-E e com o TFVF, atestando-se assim a sua validade convergente. Correlações igualmente significativas com o MoCA-E, o TS e o TFVF verificaram-se nos trabalhos de Espirito-Santo et al. (2016), de Ferreira, Lima, Peixoto e Haase (2008) e de Lima et al. (2008) em doentes portugueses com outras patologias neurológicas.

O presente estudou mostrou que a FAB discrimina a SAC da SANC de forma moderada e estatisticamente significativa, tal como em Roca et al. (2014) também numa amostra com EM. Estes resultados podem ser explicados pelos fatores potencialmente protetores que envolvem a SAC do presente estudo, tal como a reserva cognitiva alta (escolaridade elevada e profissões intelectuais) (Benedict, Morrow, Guttman, Cookfair, \& Schretlen, 2010; Valenzuela, 2019), a predominância do curso EM-RR (menos propenso a DE e cognitiva; Denney et al., 2005; Heaton et al., 1985; Smestad et al., 2010), e a toma de alguns medicamentos que podem influenciar o funcionamento cognitivo e executivo (Lacy et al., 2013; Mori et al., 2012). Tal como já referido acima, a subescala com maior poder discriminativo é a Fluência Lexical, seguida pelas Séries Motoras de Luria.

Na SAC, os resultados variam em função da escolaridade, tal como em Benke et al. (2013), Bezdicek et al. (2017) e Espirito-Santo et al. (2016) e ao contrário do que aconteceu com Lima et al. (2008). Ainda assim, a FAB demonstra ser altamente dependente do nível educacional podendo mesmo falhar em revelar a DE em pessoas altamente qualificadas (Sitek, Konkel, Dabrowska, \& Slawek, 2015). Os resultados não variaram em função do género tal como em Cohen et al. (2012) e Kim et al. (2010) nem da idade ao contrário do que se verifica nos dois estudos atrás referidos, no entanto a presente amostra é consideravelmente mais jovem.

Não se observam correlações com a idade em que foi feito o diagnóstico, ao contrário do que se observou no estudo de Smestad et al. (2010), mas que envolveu medidas da VPI, atenção e memória, para além das funções executivas. No entanto, se se considerarem as Séries Motoras de Luria, já se observa associação com o número de meses do diagnóstico, assim como discriminação entre o EM-SP e o EM-RR e EM-PP à semelhança de Smestad et al. (2010).

Em síntese, a FAB tem um desempenho psicométrico adequado, ainda que as subescalas com melhores propriedades psicométricas sejam as Fluências Lexicais e as Séries Motoras de Luria, seguidas das Semelhanças. As Fluência Lexicais e as Séries Motoras de Luria são também as mais discriminativas. À semelhança do que foi sugerido por Lima et al. (2008), a capacidade discriminativa da FAB na EM poderia aumentar ao eliminar-se ou substituir-se as subescalas do Comportamento de Preensão e das Instruções Antagónicas ou dando-Ihes menos peso para a pontuação total. Relativamente a estes resultados importa salientar que as Fluências Lexicais também medem a VPI (Bryan, Luszcz, \& Crawfard, 1997). Tendo em conta que a VPI se apresenta frequentemente alterada em doentes com EM devido aos défices na transmissão neuronal (Archibald \& Fisk, 2000; De Sonneville et al., 2002), destaca-se a importância da subescala das Fluências Lexicais para avaliar esta população. Acresce que, uma vez que Dubois et al. (2007) indicam esta subescala para o nível I da testagem da disfunção frontal na DPa, coloca-se como hipótese que aconteça o mesmo na EM.

É igualmente digno de destaque o resultado das Séries Motoras de Luria, uma vez que à semelhança do que acontece na DPa, doentes com EM apresentam problemas motores, tais como o desequilíbrio, a descoordenação e a lentificação (Feinstein, Freeman, \& Lo, 2015; Felippe et al., 2018; Johansson et al., 
2017), sendo assim de esperar que a organização, manutenção e execução de ações sucessivas se apresente alterada nesta população.

Uma limitação a este estudo poderá prender-se com o facto de não terem sido excluídos sujeitos com défice cognitivo, no entanto, esta poderá também ser considerada uma mais-valia, uma vez que este é um comprometimento comum na EM (Amato et al., 2008; Siegert \& Abernethy, 2005), permitindo, assim, uma melhor representação desta população.

Conclui-se que a FAB apresenta capacidades psicométricas e discriminativas adequadas, demonstrando ser uma ferramenta promissora para uma avaliação neuropsicológica breve e eficaz de doentes com EM. Uma ferramenta desta natureza será útil para uma orientação terapêutica adequada, assim como para o desenvolvimento de terapias de reabilitação neurocognitiva. Adicionalmente, o desempenho na subescala das Fluências Lexicais parece contribuir significativamente para as pontuações da FAB nestes doentes, colocando-se como hipótese de a incluir no nível I da avaliação neuropsicológica da disfunção frontal na EM. Seria importante em estudos futuros proceder ao cálculo da precisão diagnóstica da FAB nesta população [sensibilidade, especificidade, analise Receiver Operating Characteristic (ROC) e determinação do ponto de corte]. Nesse sentido, seria adequado determinar que doentes sofreriam ou não de défice cognitivo e, para esse efeito, poder-se-ia calcular um índice de funcionamento cognitivo recorrendo aos restantes testes neuropsicológicos e seguindo as orientações de Benedict et al. (2004). Para esse cálculo, no entanto, considerando a prevalência do diagnóstico de EM em Portugal (De Sá et al., 2006; Figueiredo et al., 2015), será necessário um número substancial de casos positivos superior para se obter poder estatístico razoável.

Por fim, recomenda-se a replicação deste estudo com uma amostra maior e mais representativa que abranja sujeitos mais velhos, menos escolarizados e com maior grau de incapacidade física e cognitiva.

\section{Agradecimentos}

Os autores agradecem à Sociedade Portuguesa de Esclerose Múltipla (SPEM) por ter autorizado a recolha da amostra clínica, aos doentes e aos profissionais envolvidos no referido recrutamento.

Conflito de interesses | Conflict of interest: nenhum | none.

Fontes de financiamento | Funding sources: nenhuma | none.

Contributos: SH: Contributo significativo na recolha e tratamento dos dados, redação do manuscrito e análise estatística. HES: Contributo significativo no desenvolvimento do desenho metodológico, análise estatística e redação e revisão do manuscrito. LC: Contributo significativo na revisão do manuscrito. LL: Contributo significativo na revisão do manuscrito. FD: Contributo significativo na revisão do manuscrito.

\section{Referências}

Alexander, M. P., \& Stuss, D. T. (2000). Disorders of frontal lobe functioning. Seminars in Neurology, 20(4), 427438. https://doi.org/10.1055/s-2000-13175

Amato, M. P., Zipoli, V., \& Portaccio, E. (2008). Cognitive changes in multiple sclerosis. Expert Review of Neurotherapeutics, 8(10), 1585-1596. https://doi.org/10.1586/14737175.8.10.1585 
Appollonio, I., Leone, M., Isella, V., Piamarta, F., Consoli, T., Villa, M., ... Nichelli, P. (2005). The Frontal Assessment Battery (FAB): Normative values in an Italian population sample. Neurological Sciences, 26(2), 108-116. https://doi.org/10.1007/s10072-005-0443-4

Archibald, C. J., \& Fisk, J. D. (2000). Information processing efficiency in patients with multiple sclerosis. Journal of Clinical and Experimental Neuropsychology, 22(5), 686-701. https://doi.org/10.1076/13803395(200010)22:5;1-9;ft686

Asaadi, S., Ashrafi, F., Omidbeigi, M., Nasiri, Z., Pakdaman, H., \& Amini-Harandi, A. (2016). Persian version of Frontal Assessment Battery: Correlations with formal measures of executive functioning and providing normative data for Persian population. Iranian Journal of Neurology, 15(1), 16-22. Retrieved from https://www.ncbi.nlm.nih.gov/pmc/articles/PMC4852066/

Benedict, R. H., Cox, D., Thompson, L. L., Foley, F., Weinstock-Guttman, B., \& Munschauer, F. (2004). Reliable screening for neuropsychological impairment in multiple sclerosis. Multiple Sclerosis Journal, 10(6), 675-678. https://doi.org/10.1191/1352458504ms1098oa

Benedict, R. H. B., Morrow, S. A., Guttman, B. W., Cookfair, D., \& Schretlen, D. J. (2010). Cognitive reserve moderates decline in information processing speed in multiple sclerosis patients. Journal of the International Neuropsychological Society, 16(5), 829-835. https://doi.org/10.1017/s1355617710000688

Benke, T., Karner, E., \& Delazer, M. (2013). FAB-D: German version of the Frontal Assessment Battery. Journal of Neurology, 260(8), 2066-2072. https://doi.org/10.1007/s00415-013-6929-8

Benton, A. L., \& Hamsher, K. (1978). Multilingual aphasia examination (MAE). Lowa City: Aja Associates.

Bezdicek, O., Růžička, F., Fendrych Mazancova, A., Roth, J., Dušek, P., Mueller, K., ... Jech, R. (2017). Frontal Assessment Battery in Parkinson's disease: Validity and morphological correlates. Journal of the International Neuropsychological Society, 23(8), 675-684. https://doi.org/10.1017/s1355617717000522

Braz, V. P. (2017). Quais as melhores letras na língua portuguesa para testar a fluência verbal fonémica e discriminar a existência e ausência de défice cognitivo? Estudo preliminar [What are the best letters in Portuguese to test phonemic verbal fluency and discriminate the existence and absence of cognitive impairment? Preliminary study]. (Master's thesis, Instituto Superior Miguel Torga, Coimbra). Retrieved from http://repositorio.ismt.pt/bitstream/123456789/712/1/Dissertação\%20Virginia.pdf

Bryan, J., Luszcz, M. A., \& Crawford, J. R. (1997). Verbal knowledge and speed of information processing as mediators of age differences in verbal fluency performance among older adults. Psychology and Aging, 12(3), 473-478.

Retrieved

from http://homepages.abdn.ac.uk/j.crawford/pages/dept/pdfs/PsychologyandAging_1997_v erbalfluency.pdf

Bugalho, P., \& Vale, J. (2011). Brief cognitive assessment in the early stages of Parkinson disease. Cognitive and Behavioral Neurology, 24(4), 169-173. https://doi.org/10.1097/wnn.0b013e3182350a1f

Chan, D., Binks, S., Nicholas, J. M., Frost, C., Cardoso, M. J., Ourselin, S., ... Chataway, J. (2017). Effect of high-dose simvastatin on cognitive, neuropsychiatric, and health-related quality-of-life measures in secondary progressive multiple sclerosis: Secondary analyses from the MS-STAT randomised, placebo-controlled trial. The Lancet Neurology, 16(8), 591-600. https://doi.org/10.1016/s1474-4422(17)30113-8

Chong, M. S., Lim, W. S., Chan, S. P., Feng, L., Niti, M., Yap, P., ... Ng, T-P. (2010). Diagnostic performance of the Chinese Frontal Assessment Battery in early cognitive impairment in an Asian population. Dementia and Geriatric Cognitive Disorders, 30(6), 525-532. https://doi.org/10.1159/000321665

Cohen, O. S., Vakil, E., Tanne, D., Molshatzki, N., Nitsan, Z., \& Hassin-Baer, S. (2012). The Frontal Assessment Battery as a tool for evaluation of frontal lobe dysfunction in patients with Parkinson disease. Journal of Geriatric Psychiatry and Neurology, 25(2), 71-77. https://doi.org/10.1177/0891988712445087

Cotter, J., Vithanage, N., Colville, S., Lyle, D., Cranley, D., Cormack, F., ... Pal, S. (2018). Investigating domainspecific cognitive impairment among patients with multiple sclerosis using touchscreen cognitive testing in routine clinical care. Frontiers in Neurology, 9(331). https://doi.org/10.3389/fneur.2018.00331

Daniel, F., Gomes, A. \& Ferreira, P. L. (2015). Contributo para a discussão da avaliação da fiabilidade de um instrumento de medição [Contributions to the discussion on the assessment of the reliability of a measurement instrument]. Revista de Enfermagem Referência, 4(7), 129-137. https://doi.org/10.12707/RIV15003

D’Onofrio, G., Panza, F., Sancarlo, D., Addante, F., Solfrizzi, V., Cantarini, C., ... Seripa, D. (2018). Executive dysfunction detected with the Frontal Assessment Battery in Alzheimer's disease versus vascular dementia. Journal of Alzheimer's Disease, 62(2), 699-711. https://doi.org/10.3233/jad-170365 
De Sá, J., Paulos, A., Mendes, H., Becho, J., Marques, J., \& Roxo, J. (2006). The prevalence of multiple sclerosis in the District of Santarém, Portugal. Journal of Neurology, 253(7), 914-918. https://doi.org/10.1007/s00415006-0132-0

De Sonneville, L. M., Boringa, J., Reuling, I. E., Lazeron, R. H., Adèr, H., \& Polman, C. (2002). Information processing characteristics in subtypes of multiple sclerosis. Neuropsychologia, 40(11), 1751-1765. https://doi.org/10.1016/s0028-3932(02)00041-6

Denney, D., Sworowski, L., \& Lynch, S. (2005). Cognitive impairment in three subtypes of multiple sclerosis. Archives of Clinical Neuropsychology, 20(8), 967-981. https://doi.org/10.1016/j.acn.2005.04.012

DeSousa, E. A., Albert, R. H., \& Kalman, B. (2002). Cognitive impairments in multiple sclerosis: A review. American Journal of Alzheimer's Disease and Other Dementias, 17(1), 23-29. https://doi.org/10.1177/153331750201700104

Dubois, B., Burn, D., Goetz, C., Aarsland, D., Brown, R. G., Broe, G. A., ... Emre, M. (2007). Diagnostic procedures for Parkinson's disease dementia: Recommendations from the movement disorder society task force. Movement Disorders, 22(16), 2314-2324. https://doi.org/10.1002/mds.21844

Dubois, B., Slachevsky, A., Litvan, I., \& Pillon, B. (2000). The FAB: A Frontal Assessment Battery at bedside. Neurology, 55(11), 1621-1626. https://doi.org/10.1212/wnl.55.11.1621

Espirito-Santo, H., \& Daniel, F. B. (2015). Calcular e apresentar tamanhos do efeito em trabalhos científicos (1): As limitações do $p<0,05$ na análise de diferenças de médias de dois grupos [Calculating and reporting effect sizes on scientific papers (1): $p<0.05$ limitations in the analysis of mean differences of two groups]. Revista Portuguesa de Investigação Comportamental e Social, 1(1), 3-16. https://doi.org/10.7342/ismt.rpics.2015.1.1.14

Espirito-Santo, H., \& Daniel, F. (2017). Calcular e apresentar tamanhos do efeito em trabalhos científicos (2): Guia para reportar a força das relações [Calculating and reporting effect sizes on scientific papers (2): Guide to report the strength of relationships]. Revista Portuguesa de Investigação Comportamental e Social, 3(1), 53-64. https://doi.org/10.7342/ismt.rpics.2017.3.1.48

Espirito-Santo, H., \& Daniel, F. (2018). Calcular e apresentar tamanhos do efeito em trabalhos científicos (3): Guia para reportar os tamanhos do efeito para análises de regressão e ANOVAs [Calculating and reporting effect sizes on scientific papers (3): Guide to report regression models and ANOVA effect sizes]. Revista Portuguesa de Investigação Comportamental e Social, 4(1), 43-60. https://doi.org/10.31211/rpics.2018.4.1.72

Espirito-Santo, H., Garcia, I. Q., Monteiro, B., Carolino, N., \& Daniel, F. (2016). Avaliação breve do défice executivo em pessoas idosas com acidente vascular cerebral: Validação da Bateria de Avaliação Frontal [Brief assessment of executive impairment in elderly with stroke: Validation of Frontal Assessment Battery]. Revista Portuguesa de Investigação Comportamental e Social, 2(2), 25-40. https://doi.org/10.7342/ismt.rpics.2016.2.2.39

Faul, F., Erdfelder, E., Lang, A. G., \& Buchner, A. (2007). G*Power 3: A flexible statistical power analysis program for the social, behavioral, and biomedical sciences. Behavior Research Methods, 39(2), 175-191. https://doi.org/10.3758/bf03193146

Feinstein, A., Freeman, J., \& Lo, A. C. (2015). Treatment of progressive multiple sclerosis: What works, what does not, and what is needed. The Lancet Neurology, 14(2), 194-207. https://doi.org/10.1016/s14744422(14)70231-5

Felippe, L. A., Salgado, P. R., Silvestre, D. S., Santos, S. M. S., \& Christofoletti, G. (2018). A controlled clinical trial on the effects of exercise on cognition and mobility in adults with multiple sclerosis. American Journal of Physical Medicine \& Rehabilitation, 98(2), 97-102. https://doi.org/10.1097/phm.0000000000000987

Ferreira, F. O., Lima, E. P., Peixoto, M. A., \& Haase, V. G. (2008). O uso de testes neuropsicológicos na esclerose múltipla e epilepsia do lobo Temporal: Relevância da estimativa de magnitude do efeito [The use of neuropsychological tests in multiple sclerosis and temporal lobe epilepsy: Relevance of the magnitude estimate of the effect]. Interamerican Journal of Psychology, 42(2), 203-217. Retrieved from http://pepsic.bvsalud.org/scielo.php?script=sci_arttext\&pid=S0034-96902008000200003\&lng=pt\&tlng=pt

Freitas, S., Simões, M. R., Martins, C., Vilar, M., \& Santana, I. (2010). Estudos de adaptação do Montreal Cognitive Assessment (MOCA) para a população portuguesa [Montreal Cognitive Assessment (MOCA) adaptation studies for the Portuguese population]. Avaliação Psicológica, 9(3), 345-357. Retrieved from http://pepsic.bvsalud.org/scielo.php? script=sci_arttext\&pid=S1677-04712010000300002 
Figueiredo, J., Silva, Â., Cerqueira, J. J., Fonseca, J., \& Pereira, P. A. (2015). MS prevalence and patients' characteristics in the district of Braga, Portugal. Neurology Research International, 2015, 1-4. https://doi.org/10.1155/2015/895163

Heaton, R. K., Nelson, L. M., Thompson, D. S., Burks, J. S., \& Franklin, G. M. (1985). Neuropsychological findings in relapsing-remitting and chronic-progressive multiple sclerosis. Journal of Consulting and Clinical Psychology, 53(1), 103-110. https://doi.org/10.1037/0022-006x.53.1.103

Hurtado-Pomares, M., Terol-Cantero, M. C., Sánchez-Perez, A., Leiva-Santana, C., Peral-Gomez, P., Valera-Gran, D., \& Navarrete-Muñoz, E. M. (2018). Measuring executive dysfunction in Parkinson's disease: Reliability and validity of the Spanish version of Frontal Assessment Battery (FAB-E). PLoS ONE, 13(11). https://doi.org/10.1371/journal. pone.0207698

HyLown Consulting LLC (2013-2019). Power and sample size [Computer Program]. Retrieved from http://powerandsamplesize.com

Jick, S. S., Li, L., Falcone, G. J., Vassilev, Z. P., \& Wallander, M.-A. (2015). Epidemiology of multiple sclerosis: Results from a large observational study in the UK. Journal of Neurology, 262(9), 2033-2041. https://doi.org/10.1007/s00415-015-7796-2

Johansson, S., Ytterberg, C., Claesson, I. M., Lindberg, J., Hillert, J., Andersson, M., ... von Koch, L. (2007). High concurrent presence of disability in multiple sclerosis. Journal of Neurology, 254(6), 767-773. https://doi.org/10.1007/s00415-006-0431-5

Jougleux-Vie, C., Duhin, E., Deken, V., Outteryck, O., Vermersch, P., \& Zéphir, H. (2014). Does fatigue complaint reflect memory impairment in multiple sclerosis? Multiple Sclerosis International, 2014, 16. https://doi.org/10.1155/2014/692468

Keegan, B. M., \& Noseworthy, J. H. (2002). Multiple sclerosis. Annual Review of Medicine, 53(1), 285-302. https://doi.org/10.1146/annurev.med.53.082901.103909

Kim, H. Y. (2013). Statistical notes for clinical researchers: Assessing normal distribution (2) using skewness and kurtosis. Restorative Dentistry \& Endodontics, 38(1), 52-54. https://doi.org/10.5395/rde.2013.38.1.52

Kim, T. H., Huh, Y., Choe, J. Y., Jeong, J. W., Park, J. H., Lee, S. B., ... Kim, K. W. (2010). Korean version of Frontal Assessment Battery: Psychometric properties and normative data. Dementia and Geriatric Cognitive Disorders, 29(4), 363-370. https://doi.org/10.1159/000297523

Kingwell, E., Marriott, J. J., Jetté, N., Pringsheim, T., Makhani, N., Marrow, S., ... Marrie, R. A. (2013). Incidence and prevalence of multiple sclerosis in Europe: A systematic review. BMC Neurology, 13(128), 1-13. https://doi.org/10.1186/1471-2377-13-128

Kratz, A. L., Braley, T. J., Foxen-Craft, E., Scott, E., Murphy, J. F., \& Murphy, S. L. (2017). How do pain, fatigue, depressive, and cognitive symptoms relate to well-being and social and physical functioning in the daily lives of individuals with multiple sclerosis? Archives of Physical Medicine and Rehabilitation, 98(11), 2160-2166. https://doi.org/10.1016/j.apmr.2017.07.004

Kugo, A., Terada, S., Ata, T., Ido, Y., Kado, Y., Ishihara, T., ... Kuroda, S. (2007). Japanese version of the Frontal Assessment Battery for dementia. Psychiatry Research, 153(1), 69-75. https://doi.org/10.1016/j.psychres.2006.04.004

Kurtzke, J. F. (2000). Multiple sclerosis in time and space-geographic clues to cause. Journal of NeuroVirology, 6(Suppl.2), S134-S140. Retrieved from https://pdfs.semanticscholar.org/d522/df170c2f692afa82c79794e82c18399dad2a.pdf

Lacy, M., Hauser, M., Pliskin, N., Assuras, S., Valentine, M. O., \& Reder, A. (2013). The effects of long-term interferon-beta-1b treatment on cognitive functioning in multiple sclerosis: A 16-year longitudinal study. Multiple Sclerosis Journal, 19(13), 1765-1772. https://doi.org/10.1177/1352458513485981

Lagarde, J., Valabrègue, R., Corvol, J.-C., Le Ber, I., Colliot, O., Vidailhet, M., \& Levy, R. (2013). The clinical and anatomical heterogeneity of environmental dependency phenomena. Journal of Neurology, 260(9), 22622270. https://doi.org/10.1007/s00415-013-6976-1

Lezak, M. D., Howieson, D. B., Bigler, E. D., \& Tranel, D. (2012). Neuropsychological assessment (5th ed). Oxford: Oxford University Press.

Lima, C. F., Meireles, L. P., Fonseca, R., Castro, S. L., \& Garrett, C. (2008). The Frontal Assessment Battery (FAB) in Parkinson's disease and correlations with formal measures of executive functioning. Journal of Neurology, 255(11), 1756-1761. https://doi.org/10.1007/s00415-008-0024-6 
Lublin, F. D., \& Reingold, S. C. (1996). Defining the clinical course of multiple sclerosis: Results of an international survey. Neurology, 46(4), 907-911. https://doi.org/10.1212/WNL.46.4.907

Lublin, F. D., Reingold, S. C., Cohen, J. A., Cutter, G. R., Sorensen, P. S., Thompson, A. J., ... Polman, C. H. (2014). Defining the clinical course of multiple sclerosis: The 2013 revisions. Neurology, 83(3), 278-286. https://doi.org/10.1212/WNL.0000000000000560

Marôco, J. (2014). Análise de Equações Estruturais: Fundamentos teóricos, software \& Aplicações (2nd ed.). Pêro Pinheiro: ReportNumber.

Matsui, H., Udaka, F., Miyoshi, T., Hara, N., Tamura, A., Oda, M., ... Kameyama, M. (2006). Frontal Assessment Battery and brain perfusion image in Parkinson's disease. Journal of Geriatric Psychiatry and Neurology, 19(1), 41-45. https://doi.org/10.1177/0891988705284714

McDonald, W. I., Compston, A., Edan, G., Goodkin, D., Hartung, H.-P., Lublin, F. D., ... Wolinsky, J. S. (2001). Recommended diagnostic criteria for multiple sclerosis: Guidelines from the international panel on the diagnosis of multiple sclerosis. Annals of Neurology, 50(1), 121-127. https://doi.org/10.1002/ana.1032

Miller, A. E. (2001). Clinical features. In S. D. Cook (Ed.), Handbook of multiple sclerosis (3rd ed.). New York: Marcel Dekker.

Milo, R., \& Miller, A. (2014). Revised diagnostic criteria of multiple sclerosis. Autoimmunity Reviews, 13(4), 518524. https://doi.org/10.1016/j.autrev.2014.01.012

Mok, V. C. T., Wong, A., Yim, P., Fu, M., Lam, W. W. M., Hui, A. C., ... Wong, K. S. (2004). The validity and reliability of Chinese Frontal Assessment Battery in evaluating executive dysfunction among Chinese patients with small subcortical infarct. Alzheimer Disease \& Associated Disorders, 18(2), 68-74. https://doi.org/10.1097/01.wad.0000126617.54783.7

Montel, S. R., \& Bungener, C. (2007). Coping and quality of life in one hundred and thirty five subjects with multiple sclerosis. Multiple Sclerosis Journal, 13(3), 393-401. https://doi.org/10.1177/1352458506071170

Mori, F., Kusayanagi, H., Buttari, F., Centini, B., Monteleone, F., Nicoletti, C. G., ... Centonze, D. (2012). Early treatment with high-dose interferon beta-1a reverses cognitive and cortical plasticity deficits in multiple sclerosis. Functional Neurology, 27(3), 163-168. $\quad$ Retrieved from https://www.ncbi.nlm.nih.gov/pmc/articles/PMC3812766/

Nasreddine, Z. S., Phillips, N. A., Bédirian, V., Charbonneau, S., Whitehead, V., Collin, I., ... Chertkow, H. (2005). The Montreal Cognitive Assessment, MoCA: A brief screening tool for mild cognitive impairment. American Geriatrics Society, 53(4), 695-699. https://doi.org/10.1111/j.1532-5415.2005.53221.x

Negreiros, A. A. L. V., Sousa-Munõz, R. L., Oliveira, B. E. S., Nóbrega, P. V., \& Monteiro, L. L. D. (2015). Clinical and epidemiological profile of patients diagnosed with multiple sclerosis in João Pessoa, Paraíba, Brazil. Arquivos de Neuro-Psiquiatria, 73(9), 741-745. https://doi.org/10.1590/0004-282×20150111

Oguro, H., Yamaguchi, S., Abe, S., Ishida, Y., Bokura, H., \& Kobayashi, S. (2006). Differentiating Alzheimer's disease from subcortical vascular dementia with the Frontal Assessment Battery test. Journal of Neurology, 253(11), 1490-1494. https://doi.org/10.1007/s00415-006-0251-7

Penny, S., Khaleeli, Z., Cipolotti, L., Thompson, A., \& Ron, M. (2010). Early imaging predicts later cognitive impairment in primary progressive multiple sclerosis. Neurology, 74(7), 545-552. https://doi.org/10.1212/WNL.0b013e3181cff6a6

Peterson, R. A. (1994). A meta-analysis of Cronbach's coefficient alpha. Journal of Consumer Research, 21(2), 381391. https://doi.org/10.1086/209405

Powell, D. J. H., Liossi, C., Schlotz, W., \& Moss-Morris, R. (2017). Tracking daily fatigue fluctuations in multiple sclerosis: Ecological momentary assessment provides unique insights. Journal of Behavioral Medicine, 40(5), 772-783. https://doi.org/10.1007/s10865-017-9840-4

Rabbitt, P. (1997). Introduction: Methodologies and models in the study of executive function. In P. Rabbitt (Ed.), Methodology of frontal and executive function (pp. 1-38). East Sussex, UK: Psychology Press Ltd.

Raimo, S., Trojano, L., Spitaleri, D., Petretta, V., Grossi, D., \& Santangelo, G. (2014). Apathy in multiple sclerosis: A validation study of the apathy evaluation scale. Journal of the Neurological Sciences, 347(1-2), 295-300. https://doi.org/10.1016/j.jns.2014.10.027

Rao, S. M. (1990). A manual for the brief, repeatable battery of neuropsychological tests in multiple sclerosis. Milwaukee: Medical College of Wisconsin. 
Roca, M., Manes, F., Gleichgerrcht, E., Ibáñez, A., González de Toledo, M. E., Marenco, V., ... Sinay, V. (2014). Cognitive but not affective theory of mind deficits in mild relapsing-remitting multiple sclerosis. Cognitive and Behavioral Neurology, 27(1), 25-30. https://doi.org/10.1097/wnn.0000000000000017

Roca, M., Torralva, T., Meli, F., Fiol, M., Calcagno, M., Carpintiero, S., ... Correale, J. (2008). Cognitive deficits in multiple sclerosis correlate with changes in fronto-subcortical tracts. Multiple Sclerosis Journal, 14(3), 364369. https://doi.org/10.1177/1352458507084270

Sarmento, L. V. S. F. (2018). Quais as melhores letras da língua portuguesa para testar a fluência verbal fonémica e discriminar a existência e ausência de défice cognitivo? Estudo de replicação [What are the best letters in the Portuguese language to test phonemic verbal fluency and discriminate the existence and absence of cognitive impairment? Replication study] (Master's thesis, Instituto Superior Miguel Torga, Coimbra). Retrieved from http://repositorio.ismt.pt/handle/123456789/953

Siegert, R. J., \& Abernethy, D. A. (2005). Depression in multiple sclerosis: A review. Journal of Neurology, Neurosurgery \& Psychiatry, 76(4), 469-475. https://doi.org/10.1136/jnnp.2004.054635

Simões, M. R., Freitas, S., Santana, I., Firmino, H., Martins, C., Nasreddine, Z., \& Vilar, M. (2008). Montreal Cognitive Assessment (MoCA): Versão portuguesa [Montreal Cognitive Assessment (MoCA): Portuguese version]. Coimbra: Serviço de Avaliação Psicológica da Faculdade de Psicologia e de Ciências da Educação da Universidade de Coimbra.

Sitek, E. J., Konkel, A., Dąbrowska, M., \& Sławek, J. (2015). Utility of Frontal Assessment Battery in detection of neuropsychological dysfunction in Richardson variant of progressive supranuclear palsy. Neurologia \& Neurochirurgia Polska, 49(1), 36-40. https://doi.org/10.1016/j.pjnns.2014.12.002

Slachevsky, A., Villalpando, J. M., Sarazin, M., Hahn-Barma, V., Pillon, B., \& Dubois, B. (2004). Frontal Assessment Battery and differential diagnosis of frontotemporal dementia and Alzheimer disease. Archives of Neurology, 61(7), 1104-1107. https://doi.org/10.1001/archneur.61.7.1104

Smestad, C., Sandvik, L., Landrø, N. I., \& Celius, E. G. (2009). Cognitive impairment after three decades of multiple sclerosis. European Journal of Neurology, 17(3), 499-505. https://doi.org/10.1111/j.14681331.2009.02889.x

Sohn-Rethel, A., \& Sohn-Rethel, M. (1978). Intellectual and manual labour: A critique of epistemology. London: Macmillan.

Thurstone, L. L. (1938). Primary mental abilities. Chicago: University of Chicago Press.

Valenzuela, M. (2019). Cognitive reserve in the aging brain. In Oxford research encyclopedia of psychology. https://doi.org/10.1093/acrefore/9780190236557.013.338

Wang, T.-L., Hung, Y.-H., \& Yang, C.-C. (2015). Psychometric properties of the Taiwanese (traditional Chinese) version of the Frontal Assessment Battery: A preliminary study. Applied Neuropsychology: Adult, 23(1), 11-20. https://doi.org/10.1080/23279095.2014.995792

Wood, B., van der Mei, I., Ponsonby, A. L., Pittas, F., Quinn, S., Dwyer, T., ... Taylor, B. (2012). Prevalence and concurrence of anxiety, depression and fatigue over time in multiple sclerosis. Multiple Sclerosis Journal, 19(2), 217-224. https://doi.org/10.1177/1352458512450351

World Health Organization. (2013). Atlas multiple sclerosis resources in the world. Geneva: WHO Press. 